\title{
Solar neutrinos: an update
}

\section{Stefano Davini*}

Department of Physics, University of Houston, Houston, TX 77204, USA

E-mail: stefano.davini@gmail.com

The Sun as a well-defined neutrino source provides extremely important opportunities to investigate nontrivial neutrino properties such as non zero mass, flavor mixing, neutrino oscillations, and MSW effect. The solar neutrino flux is energetically broadband, free of flavor backgrounds, and passes through quantities of matter obviously unavailable to terrestrial experiments. In this proceeding, after an introduction to solar neutrino phenomenology, I summarise the key features of solar neutrino experiments, then I review the recent measurements on solar neutrinos.

XIIth International Conference on Heavy Quarks \& Leptons 2014

25-29 August 2014

Schloss Waldthausen, Mainz, Germany

${ }^{*}$ Speaker. 


\section{Introduction to solar neutrinos}

The Sun is the most powerful neutrino source near the Earth. In the core of the Sun a series of nuclear reactions produce neutrinos. Two distinct processes, the main proton-proton $(p p)$ fusion chain and the sub-dominants carbon-nitrogen-oxygen (CNO) cycle, are responsible for the solar energy production. In both processes, the net effect is the fusion of four protons in a ${ }^{4} \mathrm{He}$ nucleus. Electron neutrinos $\left(v_{e}\right)$ with different energy spectra and fluxes are generated in such processes.

The main nuclear burning mechanism in the Sun is the $p p$ chain. The Carbon-NitrogenOxygen cycle (or CNO cycle), in which the fusion of four protons into a ${ }^{4} \mathrm{He}$ nucleus is achieved through reactions involving carbon, nitrogen and oxygen [1], is sub dominant for our Sun, but it is believed to be the dominant source of energy in stars more massive than about 1.3 times the mass of the Sun. The combined effect of these reactions is written as

$$
4 p \rightarrow{ }^{4} \mathrm{He}+2 e^{+}+2 v_{e} .
$$

Neutrinos that are produced within the reactions of the $p p$ chain and CNO cycle in the Sun are called solar neutrinos. The Sun is producing a great amount of neutrinos: the solar neutrino flux at the Earth distance is about $6 \times 10^{10} \mathrm{~cm}^{-2} \mathrm{~s}^{-1}$.

Physical models that describe the evolution of stars similar to our Sun are called Standard Solar Models (SSMs). Solar astrophysicists seek models that evolve in a stars with a fixed mass $M_{\odot}$, with a total luminosity $L_{\odot}$, and with an outer radius $R_{\odot}$ at an elapsed time of $4.6 \cdot 10^{9}$ years (the present age of the Sun). A Solar Model allows to determine the initial values for the mass fractions of hydrogen, helium, and heavy elements, the present distribution of physical variables inside the Sun, the spectrum of acoustic oscillation frequencies observed on the surface of the Sun, and the neutrino fluxes.

The most elaborate SSM calculations have been developed by Bahcall and his collaborators who define their SSM as the solar model which is constructed with the best available physics and input data. Therefore, their SSM calculations have been rather frequently updated. SSM's labelled as BS05(OP) [2], BSB06(GS) and BSB06(AGS) [3], BPS08(GS) and BPS08(AGS), and BPS11(GS) and BPS11(AGSS) [4] represent recent model calculations.

The measurements of the flux of the neutrinos from the Sun are used as a test to SSMs. Neutrinos are indeed the only particles which can travel undisturbed from the solar core to us, providing details about the inner workings of the Sun. Observation of solar neutrinos directly addresses the theory of stellar structure and evolution, which is the basis of the standard solar models.

More relevant for particle physics, the Sun as a well-defined neutrino source also provides important opportunities to investigate nontrivial neutrino properties such as nonzero mass, flavor mixing, neutrino oscillations, and MSW effect because of the wide range of matter density and the great distance from the Sun to the Earth. The solar neutrino flux is energetically broadband, free of flavor backgrounds, and passes through quantities of matter obviously unavailable to terrestrial experiments.

Solar neutrinos can be generated through 8 different nuclear processes. Solar neutrinos emitted in this processes are nick-named with the particles in the initial states of the reaction: $p p, p e p,{ }^{7} \mathrm{Be}$, ${ }^{8} \mathrm{~B}$, hep and the three $\mathrm{CNO}$ neutrinos ${ }^{15} \mathrm{O} v,{ }^{13} \mathrm{~N} v,{ }^{17} \mathrm{~F} v$. 
Neutrinos are emitted in the first step of the $p p$ branch through this weak interaction processes (the Q-value is written in brackets):

$$
\begin{array}{ll}
p+p \rightarrow{ }^{2} \mathrm{H}+e^{+}+v_{e} & (0.42 \mathrm{MeV}) \\
p+e^{-}+p \rightarrow{ }^{2} \mathrm{H}+v_{e} & (1.44 \mathrm{MeV})
\end{array}
$$

The reaction (1.2), called pp reaction, is the basis for the whole $p p$ chain. More than $99 \%$ of the terminations of the $p p$ chain pass though the $p p$ reaction. The rate for this primary reaction is too slow for to be measured in the laboratory at relevant energies since the reaction proceeds via the week interaction. However, the rate can be calculated accurately using the theory of low-energy weak interactions and the measured properties of the proton-proton scattering and the deuteron. The neutrino emitted in this process, called $p p$ neutrino, has a maximum energy of $0.42 \mathrm{MeV}$. The $p p$ neutrino flux predicted by the SSM has the smallest uncertainties due to the solar luminosity constraint [5].

The reaction (1.3) is called pep reaction, and the mono-energetic (1.44 MeV) neutrino emitted in this process is called pep neutrino. The pep reaction is important in principle since the ratio of pep neutrino flux to $p p$ neutrino flux is practically independent of the solar models, thus pep neutrinos contain essentially the same information about the rate of the basic fusion reactions as do the lower energy $p p$ neutrinos. The rate for the $p e p$ reaction can be calculated accurately using weak interaction theory in terms of the rate of the $p p$ reaction. The ratio between the pep and $p p$ terminations, and therefore the ratio between pep and $p p$ neutrino fluxes is $\sim 0.04$. Due to the solar luminosity constraint, the flux of pep neutrinos predicted by the SSM has the second lowest uncertainty $(1.2 \%)$ [5].

The $p p$ and pep reactions proceeds via the week interaction, so their cross sections are lower respect to strong or electromagnetic processes in the chain. Therefore $p p$ and pep reactions are a bottleneck for the $p p$ chain, and basically determine the total nuclear fusion rate and the solar luminosity.

The reaction (1.4), called hep reaction is a weak interaction process which produces the highest-energy solar neutrinos.

$$
{ }^{3} \mathrm{He}+p \rightarrow{ }^{4} \mathrm{He}+e^{+}+v_{e} \quad(19.79 \mathrm{MeV})
$$

The neutrinos from this reactions are extremely rare, and have never been detected so far.

In the Sun, ${ }^{7} \mathrm{Be}$ is almost always destroyed by electron capture, usually from free electrons in the solar plasma:

$$
{ }^{7} \mathrm{Be}+e^{-} \rightarrow{ }^{7} \mathrm{Li}+v_{e} \quad(0.862 \mathrm{MeV})
$$

The mono-energetic neutrino emitted in the electron capture of the ${ }^{7} \mathrm{Be}$ is called ${ }^{7} \mathrm{Be}$ neutrino. The rate of the process (1.5) can be calculated accurately using weak interaction theory. The ${ }^{7} \mathrm{Li}$ nucleus can be generated in an excited state with energy $487 \mathrm{keV}$, with branching factor of $10 \%$. Therefore, the energy of ${ }^{7} \mathrm{Be}$ neutrinos can be either $862 \mathrm{keV}$ or $384 \mathrm{keV}$. The flux of ${ }^{7} \mathrm{Be}$ solar neutrinos has been first measured by the Borexino experiment [6]. 
Neutrinos from ${ }^{8} \mathrm{~B}$ decay have an extremely high historical importance:

$$
{ }^{8} \mathrm{~B} \rightarrow{ }^{8} \mathrm{Be}+e^{+}+v_{e} \quad(17.98 \mathrm{MeV})
$$

High energy ${ }^{8} \mathrm{~B}$ neutrinos have been the first detected solar neutrinos in the Homestake experiment in the 60s [7]; their detection with different interaction channel in SNO led to the confirmation of solar neutrino oscillation [8][9]. The first time detection of ${ }^{8} \mathrm{~B}$ neutrinos with a $3 \mathrm{MeV}$ threshold (kinetic energy of electron recoil) has been performed in Borexino [10].

The energy production in the CNO cycle only constitutes a small contribution to the total luminosity in the Sun (of the order of $\sim 1 \%$ in the Solar Standard Models). Nevertheless, three kinds of solar neutrinos are produced in this process:

$$
\begin{aligned}
& { }^{13} \mathrm{~N} \rightarrow{ }^{13} \mathrm{C}+e^{+}+v_{e} \quad(1.19 \mathrm{MeV}) \\
& { }^{15} \mathrm{O} \rightarrow{ }^{15} \mathrm{~N}+e^{+}+v_{e} \quad(1.73 \mathrm{MeV}) \\
& { }^{17} \mathrm{~F} \rightarrow{ }^{17} \mathrm{O}+e^{+}+v_{e} \quad(1.74 \mathrm{MeV})
\end{aligned}
$$

The measurement of CNO neutrino fluxes would have important implications in solar astrophysics.

\section{Brief history of solar neutrinos}

In the past, solar neutrinos have been observed by chlorine (Homestake) and gallium (SAGE, GALLEX and GNO) radiochemical detectors and water Cherenkov detectors using light water (Kamiokande and Super-KamiokaNDE) and heavy water (SNO). Starting from 2007, low energy solar neutrinos have been successfully observed by the liquid scintillator detector Borexino.

A pioneering solar neutrino experiment by Davis and collaborators at Homestake using the ${ }^{37} \mathrm{Cl}-{ }^{37} \mathrm{Ar}$ method proposed by Pontecorvo started in the late 1960's [7]. This experiment exploited $v_{e}$ absorption on ${ }^{37} \mathrm{Cl}$ nuclei followed by the produced ${ }^{37} \mathrm{Ar}$ decay through orbital $e^{-}$capture,

$$
v_{e}+{ }^{37} \mathrm{Cl} \rightarrow{ }^{37} \mathrm{Ar}+e^{-} .
$$

The neutrino energy threshold is $814 \mathrm{keV}$. The ${ }^{37} \mathrm{Ar}$ nuclei produced are radioactive, with a half life $\left(\tau_{1 / 2}\right)$ of 34.8 days. After an exposure of the detector for two or three times $\tau_{1 / 2}$, the reaction products were chemically extracted and introduced into a low background proportional counter, where they were counted for a sufficient long period to determine the exponentially decaying signal and a constant background. Solar model calculations predict that the dominant contribution in the chlorine experiment came from ${ }^{8} \mathrm{~B}$ neutrinos, but ${ }^{7} \mathrm{Be}$, pep and $\mathrm{CNO}$ neutrinos also contributed.

From the very beginning of the solar neutrino observations, it was recognised that the observed flux was significantly smaller than the SSM predictions, provided nothing happens to the $v_{e}$ after the creation in the solar interior. This deficit has been called the Solar Neutrino problem (SNP).

Gallium experiments (GALLEX [13] and GNO [14] at Gran Sasso in Italy and SAGE [15] at Baksan in Russia) exploit the reaction

$$
v_{e}+{ }^{71} \mathrm{Ga} \rightarrow{ }^{71} \mathrm{Ge}+e^{-} .
$$

The neutrino energy threshold is $233 \mathrm{keV}$. The dominant contribution is given by the most abundant $p p$ neutrinos. Also GALLEX and SAGE detected a smaller number of neutrinos than the SSM 
predicted, but the deficit was lower with respect to the one measured by Kamiokande and SuperKamiokande.

In 1987, the KamiokaNDE experiment in Japan succeeded in real-time solar neutrino observations, exploiting $v_{e}$ elastic scattering, in a large water-Cherenkov detector. This experiment takes advantage of the directional correlation between the incoming neutrino and the recoil electron. This feature greatly helps the clear separation of the solar-neutrino signal from the background. The KamiokaNDE result gave the first direct evidence that neutrinos come from the direction of the Sun [11]. Later, the high-statistics Super-KamiokaNDE experiment [12] with a 50-kton water Cherenkov detector replaced the Kamiokande experiment. Due to high thresholds ( $7 \mathrm{MeV}$ in Kamiokande and $5 \mathrm{MeV}$ at present in Super-KamiokaNDE) the experiments observe pure ${ }^{8} \mathrm{~B}$ solar neutrinos.

The first clear evidence that solar neutrinos change flavour during the propagation from the Sun to the Earth come from the results of SNO (Sudbury Neutrino Observatory) experiment. This experiment used 100 tons of ultra-pure heavy water $\left(\mathrm{D}_{2} \mathrm{O}\right)$ contained in a spherical acrylic vessel, surrounded by an ultra-pure $\mathrm{H}_{2} \mathrm{O}$ shield. SNO measured ${ }^{8} \mathrm{~B}$ solar neutrinos via the charged-current (CC) and neutral current $(\mathrm{NC})$ reactions

$$
v_{e}+d \rightarrow e^{-}+p+p \quad(C C)
$$

and

$$
v_{x}+d \rightarrow v_{x}+p+n \quad(N C),
$$

as well as $v_{e}$ elastic scattering on electron. The $\mathrm{CC}$ reaction is sensitive only to $v_{e}$, while the NC reaction is sensitive to all active neutrinos. The results from $\mathrm{CC}$ reaction indicated significantly less flux than expected from the solar-model prediction [8], while the total flux of all neutrino flavors measured with the NC reaction agreed well with the theoretical prediction of the SSM [9]. This deficit of $v_{e}$ and the appearance of $v_{\mu}$ or $v_{\tau}$ has been understood as due to flavor conversion of solar neutrinos.

The pattern of neutrino oscillations described by Eq. (3.2) was observed by the KamLAND experiment in Japan. The neutrino oscillation parameters that are relevant in the KamLAND experiment are the same that lead to solar neutrino oscillation. KamLAND observed reactor $\bar{v}_{e}$ disappearance, with a flux-weighted average distance of $\sim 180 \mathrm{~km}$ between source and detector [17].

\section{Solar neutrino oscillations}

The existence of flavor neutrino oscillations implies that if a neutrino of a given flavor, say $v_{e}$, with energy $E$ is produced in some weak interaction process, at a sufficiently large distance $L$ from the $v_{e}$ source the probability to find a neutrino of a different flavor $\left(e . g . v_{\mu}\right)$ is different from zero.

The process of neutrino oscillation is quantum mechanical to its core, and it is a consequence of the existence of nonzero neutrino masses and neutrino (lepton) mixing.

All existing compelling data on neutrino oscillation can be described assuming 3 flavour neutrinos and 3 massive neutrino states [18]. In this description, the neutrino mixing matrix $U$ is then a $3 \times 3$ unitary matrix. The neutrino mixing matrix $U$ can be parametrised by 3 angles and $1 \mathrm{CP}$ violation phase. In order to relate the parameters to the physical observable, it is convenient to 
parametrise $U$ in such a way that the neutrino mixing angle responsible for solar (and reactor) neutrino oscillations $\theta_{12}$ and the mixing angle responsible for atmospheric neutrino oscillations $\theta_{23}$ in the 2-flavor approximation can be identified as 2 of the 3 mixing angles. The usual parametrisation of the neutrino mixing matrix $U$ is:

$$
U=\left[\begin{array}{ccc}
c_{12} c_{13} & s_{12} c_{13} & s_{13} e^{-i \delta} \\
-s_{12} c_{23}-c_{12} s_{23} s_{13} e^{i \delta} & c_{12} c_{23}-s_{12} s_{23} s_{12} e^{i \delta} & s_{23} c_{13} \\
s_{12} s_{23}-c_{12} c_{23} s_{13} e^{i \delta} & -c_{12} s_{23}-s_{12} c_{23} s_{13} e^{i \delta} & c_{23} c_{13}
\end{array}\right]
$$

where $c_{i j}=\cos \theta_{i j}, s_{i j}=\sin \theta_{i j}$, the angles $\theta_{i j}=[0, \pi / 2], \delta=[0,2 \pi]$ is the Dirac CP violation phase.

Solar neutrinos are only $v_{e}$. The survival probability $P_{e e}$, the probability that a $v_{e}$ from the Sun is detected as $v_{e}$ in the Earth, is only dependent on the mixing angles $\theta_{12}$ and $\theta_{13}$. Neglecting $\theta_{13}$, solar neutrino oscillations can be described with some degree of accuracy in a 2-neutrinos approximation. Within this approximation, $P_{e e}$ assuming propagation of neutrinos in the vacuum is:

$$
P_{e e}=1-\sin ^{2} 2 \theta_{12} \sin ^{2}\left(\frac{\Delta m_{21}^{2} L}{4 E}\right)
$$

This survival probability depends on two factors: on $\sin ^{2}\left(\Delta m_{21}^{2} L / 4 E\right)$, which exhibits oscillatory dependence on the distance $L$ and on the neutrino energy $E$ (hence the name neutrino oscillations), and on $\sin ^{2} 2 \theta_{12}$, which determines the amplitude of the oscillations. For solar neutrinos, the dimensions of the neutrino source (the Sun's core) are not negligible in comparison with the oscillation length, therefore the oscillating term is averaged out, leading to a flat, energy independent survival probability for propagation of solar neutrinos in vacuum:

$$
P_{e e}=1-\frac{1}{2} \sin ^{2} 2 \theta_{12}
$$

The approximation of solar $v_{e}$ propagation in vacuum only holds for sub $\mathrm{MeV}$ solar neutrinos, such as $p p$ neutrinos. The presence of matter can change drastically the pattern of neutrino oscillations. The $v_{e}$ and $v_{\mu}$ coherent elastic scattering on the particles of matter generates nontrivial indices of refraction of the $v_{e}$ and $v_{\mu}$ in matter [16]. The indices of refraction for $v_{e}$ and $v_{\mu}$ are different because in the ordinary matter there are no muons or muonic atoms; the difference is determined essentially by the difference of the real parts of the forward $v_{e}-e^{-}$and $v_{\mu}-e^{-}$elastic scattering amplitudes. The effect of matter in neutrino oscillations is usually called the Mikheyev, Smirnov, Wolfenstein (or MSW) effect.

The matter effects for solar neutrinos with energy less than $1 \mathrm{MeV}$, such as $p p$ neutrinos, are negligible; in this energy regime solar neutrino oscillations are described by Eq. (3.2), thus called vacuum dominated. For solar neutrinos with energy above a few $\mathrm{MeV}$, such as ${ }^{8} \mathrm{~B}$ neutrinos, matter effects are relevant, enhancing the oscillation probability; this regime of oscillation is thus called matter enhanced. In the matter enhanced regime, the survival probability is $P_{e e}=\sin ^{2} \theta_{12}$. The energy region between the vacuum dominated and matter enhanced solar $v_{e}$ oscillation regimes is called transition region. The current framework of solar neutrino oscillation, MSW-LMA, predicts a smooth transition of the $v_{e}$ survival probability between the vacuum dominated and matter enhanced regions, resulting in an energy dependent $v_{e}$ survival probability $P_{e e}$. The solar $v_{e}$ survival 




Figure 1: Survival probability of $v_{e}$ produced by the different nuclear reactions in the Sun. The vertical error bars of each data point represent the $\pm 1 \sigma$ interval; the horizontal bar shows the neutrino energy range used in the measurement. The picture is taken from [29].

probability as a function of the energy and the corresponding $P_{e e}$ measurement from solar neutrinos are shown in Fig. 1.

The net MSW effect leads again to an energy dependent survival probability for solar neutrinos. In particular, the observed deficit of solar $v_{e}$ with energy more than few $\mathrm{MeV}$ can only be explained with the MSW enhancement happening in the propagation of the neutrinos from the centre to the surface of the Sun. The MSW effect accounts for the different deficits observed by Gallex and Sage, which detected mainly $p p$ neutrinos, and Super-KamiokaNDE and SNO, which detected only ${ }^{8} \mathrm{~B}$ neutrinos.

\section{Why solar neutrino experiments are still important?}

The MSW-LMA (Large Mixing Angle) scenario is our current understanding of solar neutrino oscillations. This scenario yields to an electron neutrino survival probability $P_{e e}$ that depends on the neutrino energy. The energy dependence $P_{e e}(E)$ is predicted with high accuracy in the context of MSW-LMA oscillations and the Solar Standard Model. Any sub-leading effect in neutrino interactions like non-standard neutrino interactions (NSIs) can affect dramatically the energy dependence of $P_{e e}(E)$ [19], in particular in the transition region.

Precision measurements of low energy $p p,{ }^{7} \mathrm{Be}$, and pep solar neutrino flux are needed in order to test the MSW-LMA prediction in the vacuum-dominated and transition energy region. A measurement of the ${ }^{8} \mathrm{~B}$ solar neutrino flux with low threshold is needed to test the transition region of $P_{e e}$ between 1 and $3 \mathrm{MeV}$. A real-time experiments to allow searches for day-night asymmetries, can be used to further test the oscillation models.

Before Borexino results on ${ }^{7} \mathrm{Be}$ neutrinos, precision tests to the $P_{e e}$, thus to MSW-LMA oscillations, were only available with high energy ${ }^{8} \mathrm{~B}$ solar neutrinos, in the matter-enhanced energy 
region. Spectral measurements or high precision measurements of sub $\mathrm{MeV}$ solar neutrinos, in the vacuum-dominated energy region, were not available. Also, the detection of sub MeV solar neutrinos were only performed with gallium and chlorine radiochemical experiments, which are only sensitive the total neutrino flux above the reaction threshold. Thus, the predictions of the MSW-LMA scenario in the vacuum-dominated and transition region of the $P_{e e}$ were poorly tested.

Solar neutrino experiments are the currently the best way to measure the value of the mixing angle $\theta_{12}$. The value of the mass splitting $\Delta m_{21}^{2}$ is obtained fitting at the same time solar and reactor neutrino oscillation data, therefore any increment in the precision of solar neutrino experiments allows a more precise determination of $\theta_{12}$ and $\Delta m_{21}^{2}$.

Spectral measurements of sub-MeV solar neutrinos are also valuable for solar astrophysics. In particular, a measurement of the $\mathrm{CNO}$ neutrino flux may help solving an outstanding solar astrophysical issue known as the solar metallicity problem [4].

\section{Running solar neutrino experiments}

At present time (2014) there are three running real-time experiments which can detect solar neutrinos: the water Chereknov detector Super-KamiokaNDE and the organic liquid scintillator detectors Borexino and KamLAND. In both experiments, solar neutrinos are detected via elastic scattering with the electrons of the active mass of the detector (water or scintillator).

If the electron velocity is faster than the phase velocity of light in water, a cone on Cherenkov light is created. The Cherenkov light is projected as a ring on the wall of the detector and detected by the PMTs. Using the timing and charge information recorded by each PMT, the interaction vertex, ring direction and type of the charged particle is determined. From the sharpness of the edge of the ring the type of charged particle can be inferred. The multiple scattering of electrons is large, so electromagnetic showers produce fuzzy rings. Highly relativistic muons, in contrast, travel almost straight through the detector and produce rings with sharp edges.

Scintillator detectors have some advantages over water Cherenkov detectors. Water Cherenkov detectors, in fact, cannot effectively detect solar neutrinos whose energy is below few MeV, both because the Cherenkov light yield is low and because the intrinsic radioactive background cannot be reduced to sufficiently low levels.

An organic liquid scintillator solves the aforementioned problems: the low energy neutrino detection is possible because of the high light yield that in principle allow the energy threshold to be set down to a level of a few tens of $\mathrm{keV}^{1}$. The organic nature of the scintillator, and its liquid form at the ambient temperature, provide very low solubility of ions and metal impurities, and yield the technical possibility to purify the material as requested. However, being the scintillator light emitted isotropically, no measurement of the direction of the incoming neutrino is possible and single neutrino induced events are intrinsically indistinguishable from $\beta$ and $\gamma$ radioactivity, posing formidable requirements in terms of radiopurity of the scintillator and of the detector materials.

\footnotetext{
${ }^{1}$ However, the unavoidable contamination of ${ }^{14} \mathrm{C}$ that is present in any organic liquid practically limits the neutrino detection window above $\sim 200 \mathrm{keV}$.
} 


\subsection{Super-KamiokaNDE}

Super-KamiokaNDE (abbreviated to Super-K or SK) is a neutrino observatory located under Mount Kamioka in Japan. The observatory was designed to search for proton decay, study solar, atmospheric and accelerator neutrinos [20].

The Super-K is located $1000 \mathrm{~m}$ underground in the Mozumi Mine in Hida's Kamioka area. It consists of a cylindrical stainless steel tank that is $41.4 \mathrm{~m}$ tall and $39.3 \mathrm{~m}$ in diameter holding 50,000 tons of ultra-pure water. The tank volume is divided by a stainless steel superstructure into an inner detector (ID) region that is $33.8 \mathrm{~m}$ in diameter and $36.2 \mathrm{~m}$ in height and outer detector (OD) which consists of the remaining tank volume. Mounted on the superstructure are 11146 photomultiplier tubes (PMT) that face the ID and 1885 PMTs that face the OD.

Super-K takes advantage of the directional correlation between the incoming neutrino and the recoil electron to discriminate the solar-neutrino signal from the background.

\subsection{Borexino}

Borexino is a large volume liquid scintillator detector [21]. It is located deep underground ( $\sim 3800 \mathrm{~m}$ of water equivalent) in the Hall $\mathrm{C}$ of the Laboratori Nazionali del Gran Sasso (LNGS) in Italy. The main goal of the experiment is the detection of low energy solar neutrinos, such as the ${ }^{7} \mathrm{Be}$, pep, and CNO solar neutrinos.

In Borexino low energy neutrinos of all flavors are detected by means of their elastic scattering off electrons. The electron recoil energy is converted into scintillation light which is then collected by a set of 2212 photomultipliers (PMTs). Borexino has an active mass of 278 tons of pseudocumene scintillator ( $\mathrm{PC}, 1,2$,4-trimethylbenzene $\mathrm{C}_{6} \mathrm{H}_{3}\left(\mathrm{CH}_{3}\right)_{3}$ ), doped with $1.5 \mathrm{~g} / \mathrm{l}$ of flour PPO (2, 5-trimethylbenzene, $\mathrm{C}_{15} \mathrm{H}_{11} \mathrm{NO}$ ) as a solute.

The scintillator is contained in a thin $(125 \mu \mathrm{m})$ nylon vessel and is surrounded by two concentric PC buffers (323 and 567 tons) doped with $5.0 \mathrm{~g} / \mathrm{l}$ of DMP (dimethylphthalate) that is added as a light quencher in order to further reduce the scintillation yield of pure PC. The two PC buffers are separated by a second thin nylon membrane to prevent ${ }^{222} \mathrm{Rn}$ emanated from the external materials to diffuse towards the scintillator. The scintillator and buffers are contained in a stainless steel sphere with diameter $13.7 \mathrm{~m}$.

\subsection{KamLAND}

The Kamioka Liquid Scintillator Antineutrino Detector (KamLAND) is built at the Kamioka Observatory, an underground neutrino detection facility near Toyama, Japan. The detector is situated in a drift mine shaft in the old KamiokaNDE cavity in the Japanese Alps. The site is surrounded by 53 Japanese commercial nuclear reactors. KamLAND is located at an average fluxweighted distance of approximately 180 kilometers from the reactors, which makes it sensitive to the mixing of neutrinos associated with LMA oscillation parameters.

The KamLAND detector's outer layer consists of an 18 meter-diameter stainless steel containment vessel with an inner lining of 1879 PMTs, each 50 centimetres in diameter. Its second, inner layer consists of a $13 \mathrm{~m}$-diameter nylon balloon filled with a liquid scintillator composed of 1,000 metric tons of mineral oil, benzene, and fluorescent chemicals. Non-scintillating, highlypurified oil provides buoyancy for the balloon and acts as a buffer to keep the balloon away from 
the photo-multiplier tubes; the oil also shields against external radiation. A 3.2 kiloton cylindrical water Cherenkov detector surrounds the containment vessel, acting as a muon veto counter and providing shielding from cosmic rays and radioactivity.

Compared to Borexino, KamLAND has a larger detection volume but worse radiopurity, hence more background.

\section{Update on ${ }^{8} \mathbf{B}$ neutrino measurements}

${ }^{8} \mathrm{~B}$ solar neutrinos have been detected by Super-KamiokaNDE, with an energy threshold down to $4.5 \mathrm{MeV}$ in its final phase, by SNO exploiting elastic scattering, charged and neutral current interactions, by KamLAND, with a threshold of $5 \mathrm{MeV}$, and by Borexino with an energy threshold down to $3 \mathrm{MeV}$ but smaller statistics compared to the other experiments. A day-night asymmetry in the interaction rate has been evidenced by Super-KamiokaNDE. However, there is no evidence so far of the $P_{e e}$ increase below $6 \mathrm{MeV}$ predicted by the MSW-LMA oscillation.

\subsection{Super-K ${ }^{8}$ B rate}

Super-KamiokaNDE-IV (SK-IV) data taking began in September of 2008, after upgrading the electronics and data acquisition system [22]. Due to these upgrades and improvements to water system dynamics, calibration and analysis techniques, a solar neutrino signal could be extracted at recoil electron kinetic energies as low as $3.5 \mathrm{MeV}$. When the SK-IV data is combined with the previous three SK phases, the SK extracted solar neutrino flux is found to be $(2.37 \pm 0.015$ (stat) \pm 0.04 (syst) $) \times 10^{6} \mathrm{~cm}^{-2} \mathrm{sec}^{-1}$.

The combination of the SK recoil electron energy spectra slightly favours distortions due to a changing electron flavor content. Such distortions are predicted when assuming solar neutrino oscillation described by MSW-LMA. A solar neutrino global oscillation analysis including all current solar neutrino data, as well as KamLAND reactor antineutrino data, measures the solar mixing angle as $\sin ^{2} \theta_{12}=0.305 \pm 0.013$, the solar neutrino mass squared splitting as $\Delta m_{21}^{2}=7.49_{-0.17}^{+0.19} \times 10^{-5} \mathrm{eV}^{2}$ and $\sin ^{2} \theta_{13}=0.026_{-0.012}^{+0.017}$.

\subsection{Super-K ${ }^{8}$ B day-night asymmetry}

Super-KamiokaNDE reported in 2014 an indication that the elastic scattering rate of solar ${ }^{8} \mathrm{~B}$ neutrinos with electrons in the Super-K detector is larger when the neutrinos pass through the Earth during nighttime [23]. Super-K determines the day-night asymmetry, defined as the difference of the average day rate and average night rate divided by the average of those two rates, to be $(-3.2$ \pm 1.1 (stat) \pm 0.5 (syst) $) \%$, which deviates from zero by $2.7 \sigma$.

Since the elastic scattering process is mostly sensitive to electron- flavored solar neutrinos, a non-zero day-night asymmetry implies that the flavor oscillations of solar neutrinos are affected by the presence of matter within the neutrinos' flight path. Super-KamiokaNDE's day/night asymmetry is consistent with neutrino oscillations for $4 \times 10^{-5} \mathrm{eV}^{2}<\Delta m_{21}^{2}<7 \times 10^{-5} \mathrm{eV}^{2}$ and large mixing values of $\theta_{12}$, at the $68 \%$ C.L. 


\section{3 $\mathrm{SNO}^{8} \mathrm{~B}$ rate}

SNO reported in 2011 results from a combined analysis of solar neutrino data from all phases of the Sudbury Neutrino Observatory [24]. By exploiting particle identification information obtained from the proportional counters installed during the third phase, the analysis improved background rejection in that phase of the experiment. The combined analysis resulted in a total flux of active neutrino flavors from ${ }^{8} \mathrm{~B}$ decays in the Sun of $(5.25 \pm 0.16$ (stat) $+0.11-0.13$ (syst) $) \times$ $10^{6} \mathrm{~cm}^{-2} \mathrm{~s}^{-1}$.

A two-flavor neutrino oscillation analysis yielded $\Delta m_{21}^{2}=\left(5.6_{-1.4}^{+1.9}\right) \times 10^{-5} \mathrm{eV}^{2}$ and $\tan ^{2} \theta_{12}=$ $0.427_{-0.029}^{+0.033}$. A three-flavor neutrino oscillation analysis combining this result with results of all other solar neutrino experiments and the KamLAND experiment yielded $\Delta m_{21}^{2}=\left(7.41_{-0.19}^{+0.21}\right) \times$ $10^{-5} \mathrm{eV}^{2}, \tan ^{2} \theta_{12}=0.446_{-0.029}^{+0.030}$, and $\sin ^{2} \theta_{13}=\left(2.5_{-1.5}^{+1.8}\right) \times 10^{-2}$. This implied an upper bound of $\sin ^{2} \theta_{13}<0.053$ at the $95 \%$ confidence level (C.L.).

\subsection{KamLAND ${ }^{8}$ B rate}

KamLAND reported in 2011 a measurement of the neutrino-electron elastic scattering rate from ${ }^{8} \mathrm{~B}$ solar neutrinos based on a 123 kton-day exposure [25]. The background-subtracted electron recoil rate, above a $5.5 \mathrm{MeV}$ analysis threshold is $1.49 \pm 0.14$ (stat) \pm 0.17 (syst) events per kton-day. Interpreted as due to a pure electron flavor flux with a ${ }^{8} \mathrm{~B}$ neutrino spectrum, this corresponds to a spectrum integrated flux of $2.77 \pm 0.26$ (stat) \pm 0.32 (syst) $\times 10^{6} \mathrm{~cm}^{-2} \mathrm{~s}^{-1}$. The measured rate is consistent with existing measurements and with Standard Solar Model predictions which include matter enhanced neutrino oscillation.

The analysis threshold is driven by ${ }^{208} \mathrm{Tl}$ present in the liquid scintillator, and the main source of systematic uncertainty is due to background from cosmogenic ${ }^{11} \mathrm{~B}$.

\subsection{Borexino ${ }^{8} \mathrm{~B}$ rate}

Borexino reported in 2010 the measurement of electron neutrino elastic scattering from ${ }^{8} \mathrm{~B}$ solar neutrinos with $3 \mathrm{MeV}$ energy threshold [10]. The rate of solar neutrino-induced electron scattering events above this energy in Borexino is $0.217 \pm 0.038$ (stat) \pm 0.008 (syst) cpd $/ 100$ ton, which corresponds to the equivalent unoscillated flux of $(2.4 \pm 0.4$ (stat) \pm 0.1 (syst) $) \times 10^{6} \mathrm{~cm}^{-2} \mathrm{~s}^{-1}$, in good agreement with measurements from SNO and Super-KamiokaNDE. Assuming the ${ }^{8} \mathrm{~B}$ neutrino flux predicted by the high metallicity Standard Solar Model, the average ${ }^{8} \mathrm{~B}$ neutrino survival probability above $3 \mathrm{MeV}$ is measured to be $0.2 \pm 0.1$. Borexino's results are consistent with the prediction of the MSW-LMA solution of a transition in the solar electron neutrino survival probability between the low energy vacuum-driven and the high-energy matter-enhanced solar neutrino oscillation regimes.

\section{Update on ${ }^{7}$ Be neutrino measurements}

The $862 \mathrm{keV}^{7} \mathrm{Be}$ solar neutrino rate has been measured precisely by the Borexino experiment. Borexino also reported a null result in the ${ }^{7} \mathrm{Be}$ day-night asymmetry. Recently, also KamLAND reported its first measurement of ${ }^{7} \mathrm{Be}$ neutrinos. 


\subsection{Borexino ${ }^{7}$ Be rate}

Borexino reported in 2011 a precise direct measurement of the $862 \mathrm{keV}{ }^{7} \mathrm{Be}$ solar neutrino interaction rate yielding $46.0 \pm 1.5(\mathrm{stat})_{-1.5}^{+1.6}$ (syst) counts/day/(100 tons) [6]. This corresponds to $v_{e}$ equivalent ${ }^{7} \mathrm{Be}$ solar neutrino flux of $(3.10 \pm 0.15) \times 10^{9} \mathrm{~cm}^{-2} \mathrm{~s}^{-1}$ and, under the assumption of $v_{e}$ transition to other active neutrino flavours, yields an electron neutrino survival probability of $0.51 \pm 0.07$ at $862 \mathrm{keV}$. The no flavor change hypothesis is ruled out at $5 \sigma$. These results significantly improve the precision with which the MSW-LMA neutrino oscillation model is experimentally tested at low energy.

\subsection{Borexino ${ }^{7}$ Be day-night asymmetry}

Borexino reported in 2012 the null result of a search for a day-night asymmetry in the ${ }^{7} \mathrm{Be}$ solar neutrino interaction rate [26]. The measured asymmetry is $A_{d n}=0.001 \pm 0.012$ (stat) \pm 0.007 (syst), in agreement with the prediction of MSW-LMA solution for neutrino oscillations. This result disfavours MSW oscillations with mixing parameters in the LOW region at more than $8.5 \sigma$.

This region is, for the first time, strongly disfavoured without the use of reactor anti-neutrino data and therefore the assumption of CPT symmetry. The result can also be used to constrain some neutrino oscillation scenarios involving new physics.

\subsection{KamLAND ${ }^{7}$ Be rate}

KamLAND reported in 2014 a measurement of the $v_{e}$ elastic scattering rate of $862 \mathrm{keV}{ }^{7} \mathrm{Be}$ solar neutrinos based on a 165.4 kton-day exposure [27]. The observed rate is $582 \pm 90$ (kton $\times$ day $)^{-1}$, which corresponds to a $862 \mathrm{keV}^{7} \mathrm{Be}$ solar neutrino flux of $(3.26 \pm 0.50) \times 10^{9} \mathrm{~cm}^{-2} \mathrm{~s}^{-1}$, assuming a pure electron flavor flux. Comparing this flux with the standard solar model prediction and further assuming three flavor mixing, a $v_{e}$ survival probability of $(0.66 \pm 0.14)$ is determined from the KamLAND data. Using a global three flavor oscillation analysis, KamLAND obtains a total ${ }^{7} \mathrm{Be}$ solar neutrino flux of $(5.82 \pm 0.98) \times 10^{9} \mathrm{~cm}^{-2} \mathrm{~s}^{-1}$, which is consistent with the standard solar model predictions.

\section{Update on pep and CNO neutrino measurements}

Borexino reported in 2012 the first time direct observation of solar neutrinos in the $1.0-1.5$ $\mathrm{MeV}$ energy range [28]. Borexino measured the rate of pep solar neutrino interactions in Borexino to be $(3.1 \pm 0.6$ (stat) \pm 0.3 (syst) ) counts/(day x 100 ton) and provided a constraint on the CNO solar neutrino interaction rate of $<7.9$ counts /(day x 100 ton) (95\% C.L.).

The absence of the solar neutrino signal is disfavoured at $99.997 \%$ C.L., while the absence of the pep signal is disfavored at $98 \%$ C.L. The no flavor change hypothesis for pep neutrinos is ruled out at $97 \%$. Assuming the MSW-LMA solution to solar neutrino oscillations, these values correspond to solar neutrino fluxes of $(1.6 \pm 0.3) \times 10^{8} \mathrm{~cm}^{-2} \mathrm{~s}^{-1}$ and $7.7 \times 10^{8} \mathrm{~cm}^{-2} \mathrm{~s}^{-1}(95 \%$ C.L.), respectively, in agreement with the Standard Solar Model.

These results represent the first measurement of the pep neutrino flux and the strongest constraint of the CNO solar neutrino flux to date. This unprecedented sensitivity was achieved by adopting novel data analysis techniques for the rejection of cosmogenic ${ }^{11} \mathrm{C}$, the dominant background in the $1-2 \mathrm{MeV}$ region. 


\section{Update on $p p$ neutrino measurements}

Borexino reported in 2014 the first time spectral measurement of $p p$ solar neutrinos [29]. The solar $p p$ neutrino interaction rate measured by Borexino is $144 \pm 13$ (stat) \pm 10 (syst) cpd per 100 ton. The absence of $p p$ solar neutrinos is excluded with a statistical significance of $10 \sigma$. Using the latest values of the neutrino oscillation parameters, the measured solar $p p$ neutrino flux is $(6.6 \pm 0.7) \times 10^{10} \mathrm{~cm}^{-2} \mathrm{~s}^{-1}$ in good agreement with the prediction of the standard solar model $\left(5.98 \times(1 \pm 0.006) \times 10^{10} \mathrm{~cm}^{-2} \mathrm{~s}^{-1}\right)$. The survival probability is found to be $P_{e e}=0.64 \pm 0.12$, providing a constraint of the MSW-LMA oscillation model in the low energy vacuum regime.

The observation of $p p$ neutrinos provides us with a direct glimpse at the keystone fusion process that keeps the Sun shining, showing that about $99 \%$ of the power of the Sun $\left(3.84 \times 10^{33} \mathrm{ergs}\right.$ per second) is generated by the proton-proton fusion process.

\section{Conclusions and outlook}

Solar neutrinos have been pivotal to the discovery of neutrino oscillations; still it is a very active field of research. The study of the solar spectrum has progressed a lot, however CNO solar neutrino detection is still missing. The MSW-LMA effect is observed, but still with relatively large errors (see Figure 1). In particular, there is no evidence yet of the upturn of $P_{e e}$ in the low energy part of ${ }^{8} \mathrm{~B}$ neutrino spectrum.

The next goal in this area of research are precision measurements of solar neutrino rates and day-night asymmetries that will probe $P_{e e}$ and constrain non-standard neutrino and solar physics. The high precision era of solar neutrino physics is coming.

\section{References}

[1] J.N. Bahcall, Neutrino Astrophysics (Cambridge University Press, 1989)

[2] J.N. Bahcall, A.M. Serenelli, S. Basu, Astrophys. J. Suppl 621, (2005) L85.

[3] J.N. Bahcall, A.M. Serenelli, S. Basu, Astrophys. J. Suppl 165, (2006) 400.

[4] A. Serenelli, W. Haxton, C. Pena-Garay, The Astrophysical Journal 743, (2011) 24. S. Basu, Proc. GONG 2008/SOHO XXI Solar-stellar dynamos as revealed by helio and asteroseismology, edited by Mausumi Dikpati et al., Vol. 416 (2009) 193.

[5] J.N. Bahcall, Phys. Rev. C 65, (2002) 025801.

[6] G. Bellini et al., Phys. Rev. Lett. 107, (2011) 141302. DOI: 10.1103/PhysRevLett.107.141302.

[7] R. Davis R, D.S. Harmer, K.C. Hoffman, Phys. Rev. Lett. 20, (1968) 1205.

[8] Q.R. Ahmad et al., Phys. Rev. Lett. 87, (2001) 071301.

[9] Q.R. Ahmad et al., Phys. Rev. Lett. 89, (2002) 011301.

[10] G. Bellini et al., Phys. Rev. D 82, (2010) 033006. DOI: 10.1103/PhysRevD.82.033006

[11] K.S. Hirata et al., Phys. Rev. Lett. 63, (1989) 16.

[12] J.P. Cravens et al., Phys. Rev. D 78, (2008) 032002. DOI: 10.1103/PhysRevD.78.032002. 
[13] W. Hampbel et al., Phys. Lett. B 447, (1999) 127.

[14] M. Altman et al., Phys. Lett. B 616, (2005) 174.

[15] A. Abdurashitov et al., Phys. Rev. C 80, (2009) 015807.

[16] L. Wolfenstein, Phys. Rev. D 17, (1978) 2369.

[17] K. Eguchi et al., Phys. Rev. Lett. 90, (2003) 021802.

[18] J. Beringer et al. (Particle Data Group), Phys. Rev. D 86, (2012) 010001.

[19] A. Friedland et al., Phys. Lett. B 594, (2004) 347.

[20] S. Fukuda; et al., Nucl. Instr. and Meth. A 501, (2003) $418 Đ 462$.

[21] G. Alimonti et al., Nucl. Instr. and Meth. A 600, (2009) 568.

[22] A. Renshaw for the Supe-KamiokaNDE Collaboration, arXiv:1403.4575 [hep-ex]

[23] A. Renshaw et al. (the Super-KamiokaNDE Collaboration), Phys. Rev. Lett. 112, (2014) 091805

[24] B. Aharmim et al. (the SNO Collaboration), Phys. Rev. C 88, (2013) 02550

[25] S. Abe et al. (KamLAND Collaboration), Phys. Rev. C 84, (2011) 035804

[26] G. Bellini et al. (the Borexino Collaboration), Phys. Lett. B 707, (2012) 22.

[27] KamLAND Collaboration, arXiv:1405.6190 [hep-ex]

[28] G. Bellini et al. (the Borexino Collaboration), Phys. Rev. Lett. 108, (2012) 051302. doi: 10.1103/PhysRevLett.108.051302.

[29] G. Bellini et al. (the Borexino Collaboration), Nature 512, 383-386 (2014) doi:10.1038/nature13702 